\title{
APROXIMACIÓN EN EL COMPORTAMIENTO DE LOS SUELOS DEL CAMPO DE ALICANTE A UNA LEY FÍSICA DE INFILTRACIÓN
}

\author{
Concepción Bru Ronda y Artemio Cuenca Payá
}

\section{RESUMEN}

A tenor de las pruebas de infiltración realizadas en el área del Campo de Alicante, se ha estudiado dicho proceso en estado transitorio. Asimilando éste a un proceso de propagación térmica se obtiene por separado una estimación del coeficiente de permeabilidad y del coeficiente de difusividad del suelo que recibe la precipitación.

\begin{abstract}
From testa of infiltration carried out in the area of the «Campo de Alicante» we have studied the transitory state of the process. Assimilating it to a model of thermical propagation we obtain separately an estimation of the coefficient of permeability and the coefficient of diffusivity.
\end{abstract}

\section{Introducción}

En un trabajo anterior, los autores analizaron el comportamiento de los suelos del Campo de Alicante durante los primeros segundos del proceso en régimen transitorio (Bru Ronda y Cuenca Payá, 1986), que posteriormente ampliaron al régimen estacionario para definir las tasas de infiltración final (Gil Olcina et Alter, 1986). 
Ahora se han comparado las curvas de velocidad de infiltración obtenidas en suelos homogéneos con una función de infiltración de «tipo Philip» de la forma:

$$
I(t)=A+B t^{-1 / 2}
$$

presentando la posibilidad de determinar los coeficientes de permeabilidad (K) y de difusividad (C) a partir de un símil basado en la propagación térmica desde una superficie hacia un semiespacio.

\section{Discusión}

De los 21 puntos estudiados inicialmente, sólo 9 cumplen condiciones de homogeneidad en el perfil como para que éste pueda ser asimilado a un semiespacio. Comparando los valores reales obtenidos, con una función de la fórmula (1) se obtienen los resultados de la Tabla I, en la que $A$ y $B$ son los parámetros de aquella función y $r$ el coeficiente de correlación. El número que identifica a cada ensayo se refiere a su localización en el segundo trabajo y que ha sido indicada en el mapa 1.

\section{TABLA I}

\begin{tabular}{cccc} 
Ensayo $\mathrm{n}^{\circ}$ & $\mathrm{A}$ & $\mathrm{B}$ & $\mathrm{r}$ \\
\hline 1 & 0.0022 & 5.4889 & 0.952 \\
2 & 0.0194 & 0.2253 & 0.994 \\
3 & 0.0119 & 1.2854 & 0.998 \\
8 & 0.0198 & 13.8332 & 0.980 \\
9 & 0.0621 & 22.5643 & 0.996 \\
13 & 0.0250 & 0.2324 & 0.975 \\
15 & 0.0036 & 0.2620 & 0.985 \\
17 & 0.0332 & 1.9428 & 0.959 \\
20 & 0.0090 & 0.2511 & 0.992
\end{tabular}

El volumen infiltrado al cabo de un tiempo $t$ se obtiene por integración de (1), resultando:

$$
V_{I}=A t+2 B \sqrt{t}
$$

en la que el término $A t$ domina durante el régimen estacionario, mientras que el segundo lo hace en el transitorio.

Utilizando una analogía térmica (Kreithy Black, 1983:142) se puede poner para el régimen transitorio:

$$
I(t)=\frac{K \Delta w}{\sqrt{\pi c t}}
$$


que integrada al tiempo $t$ da el volumen infiltrado:

$$
V_{I}=2 k \Delta w \sqrt{\frac{t}{\pi c}}
$$

siendo:

$\mathrm{K}=$ Conductividad $(=$ Permeabilidad $)(\mathrm{cm} / \mathrm{seg})$.

$\mathrm{c}=$ Difusividad $\left(\mathrm{cm}_{2} / \mathrm{seg}\right)$.

$\mathrm{w}=$ Variación de la humedad hasta saturación ( $\%$ en volumen $)$.

Igualando (3) y (4) se obtiene: $B=\frac{K \Delta w}{\sqrt{c}}$ (5), de la que puede deducirse que los coeficientes de permeabilidad (K) y difusión (c) se encuentran relacionados mediante: (6)

$$
\frac{k}{\sqrt{c}}=\frac{B \backslash T T}{\Delta w}
$$

De (5) se deduce que en la zona ya saturada se cumple que $\mathrm{B}=0$, con lo que sólo es significativo el término $A t$. de forma que en régimen estacionario el volumen infiltrado es:

$\mathrm{Ve}=\mathrm{At}=\mathrm{i \lambda Kt}(\mathrm{7})$.

El parámetro $\lambda$, que caracteriza la filtración no saturada, es próximo a cero en régimen transitorio, pero vale la unidad cuando se cumple (7), por tanto $A=i K(8)$, de la que puede deducirse el valor de $K$ a partir de $A$ y del gradiente $i$ y el de $c$ a partir de (6) conociendo $K$ y el intervalo $\Delta \mathrm{w}$.

Los valores de $\Delta w$ para los suelos estudiados oscilan entre aproximadamente 30 y 50 , por lo que puede tomarse un valor medio de cálculo de $\Delta \mathrm{w} \simeq 40$, mientras que el gradiente hidráulico medio durante los ensayos es de 20, por lo que aplicando (8) y (6) se obtienen los valores de $K$ y $c$ presentados en la Tabla II, junto a las características texturales del suelo y el valor de la infiltración final $\left(\mathrm{I}_{\mathrm{F}}\right)$ determinado en campo.

\section{TABLA II}

$\begin{array}{ccccl}\text { Ensayo } \mathrm{n}^{\circ} & \mathrm{K}(\mathrm{cm} / \mathrm{seg}) & \mathrm{C}\left(\mathrm{cm}^{2} / \mathrm{seg}\right) & \mathrm{I}_{\mathrm{F}}(\mathrm{cm} / \mathrm{seg}) & \text { Tipo de suelo } \\ 1 & 1.1 .10^{-4} & 2.0 .10^{-7} & 0,0092 & \text { Alteración sustrato margoso } \\ 2 & 9.7 .10^{-4} & 9.4 \cdot 10^{-3} & 0,0300 & \text { Litosuelo sobre karst } \\ 3 & 6.0 .10^{-4} & 1.1 .10^{-4} & 0,0230 & \text { Arenas limosas } \\ 8 & 9.9 .10^{-4} & 3,0.10^{-6} & 0,0020 & \text { Margas meteorizados } \\ 9 & 3.1 .10^{-3} & 1,0.10^{-5} & 0.0027 & \text { Limos arcillosos } \\ 13 & 1.3 .10^{-3} & 1,5.10^{-2} & 0,0270 & \text { Aluvión con gravas } \\ 15 & 1.8 .10^{-4} & 2,4.10^{-4} & 0,0120 & \text { Margas fisuradas y alteradas } \\ 17 & 1.7 .10^{-3} & 3.7 .10^{-4} & 0.0100 & \text { Limo arenoso } \\ 20 & 4.5 \cdot 10^{-4} & 1,6.10^{-3} & 0.0100 & \text { Arena arcillosa }\end{array}$




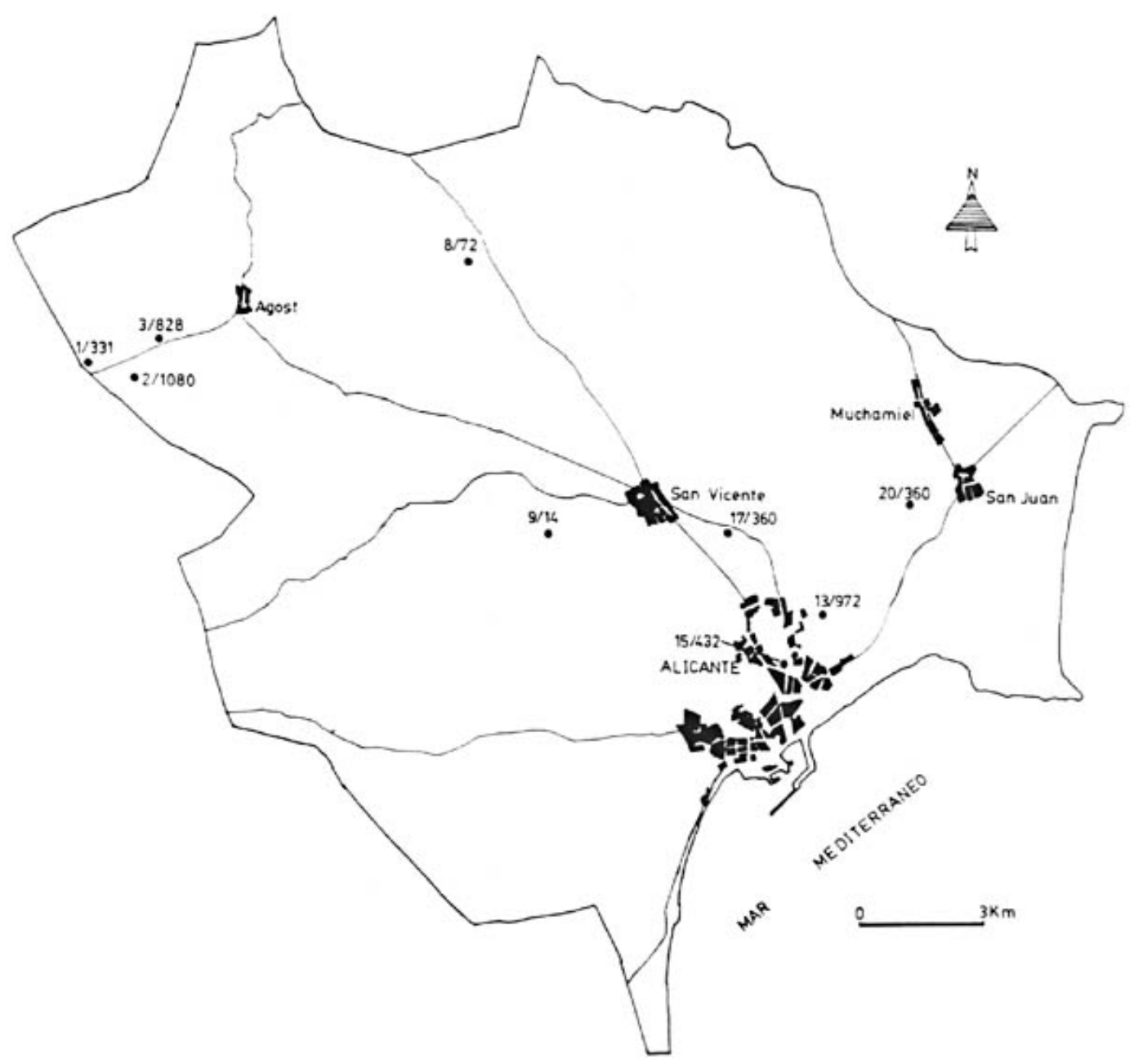

Mapa 1: Localización de las pruebas de infiltración.

\section{Conclusiones}

De lo expuesto se deduce que los datos experimentales se aproximan a una ley física de infiltración de la forma propuesta por Philip:

$$
I(t)=A+B t^{-1 / 2}
$$

que permite estimar los coeficientes de permeabilidad y de difusión siempre que la capa de suelo pueda asimilarse a un semiespacio homogéneo. Los valores de $K$ obtenidos son coherentes con las características texturales de los suelos, quedando dentro de los órdenes de magnitud que podría esperarse (Vidal Pardal, 1962), mientras que los coeficientes de difusión muestran una cierta correlación con los valores 
de $\mathrm{I}_{\mathrm{F}}$ según una ecuación de la forma:

$$
\mathrm{I}_{\mathrm{F}}=0,0097+1,4286 \mathrm{c}
$$

con un coeficiente de correlación:

$$
\mathrm{r}=0,760
$$

Esto, que en un principio puede parecer una contradicción, se explica por el hecho de que los ensayos se realizaron para estudiar la infiltración durante aguaceros de corta duración (10 minutos), y el $\mathrm{I}_{\mathrm{F}}$ determinado experimentalmente se encuentra fuertemente influenciado todavía por los procesos en régimen transitorio.

Como comentario final se hace necesario señalar que los ensayos realizados con infiltrómetros sobrevaloran la capacidad de infiltración del suelo durante un aguacero, ya que en este caso el fluido que percola no es agua pura, sino una suspensión más o menos concentrada de partículas finas y ultrafinas con viscosidad superior a la del agua, por lo que en los modelos teóricos debe introducirse un «término de viscosidad» que cuantifique ese fenómeno, siendo este el objeto de las investigaciones que actualmente llevan a cabo los autores.

\section{BIBLIOGRAFÍA}

BRU RONDA, C. y CUENCA PAYÁ, A., «Primeras evaluaciones de la capacidad de infiltración en suelos de la comarca del Campo de Alicante». Estudios sobre Geomorfología del Sur de España. Departamento de Geografía Física de la Universidad de Murcia. Murcia, 1986. Pp. 27-31.

GIL OLCINA, A. el ALT., Inundaciones en la Ciudad y Término de Alicante. Universidad de Alicante-Ayuntamiento de Alicante. Alicante, 1986. 179 páginas.

KREITH, F. y BLACK, W. Z., La transmisión del calor. Principios fundamentales. Alhambra Universidad. Madrid, 1983. 544 pp.

VIDAL PARDAL, M., Estudio acerca de la permeabilidad del terreno. Servicio Geológico del Ministerio de Obras Públicas. Boletín n ${ }^{\circ} 15$ (Información y Estudios). Madrid, 1962, 66 pp. 